\title{
How Social Norms Promote Misleading Social Feedback and Inaccurate Self-Assessment
}

\author{
Adam J. Fay ${ }^{1}$, Alexander H. Jordan ${ }^{2}$, and Joyce Ehrlinger ${ }^{1}$ * \\ 1 Florida State University \\ 2 Dartmouth College
}

\begin{abstract}
Self-assessments are often prone to error. Past research has identified cognitive and motivational biases that lead self-assessments astray. In the present paper, we discuss how behavior shaped by social norms leaves the negative information that people require for accurate self-assessments invisible. First, social norms lead people to suppress critical feedback in favor of more positive evaluations. Although people recognize that they prefer to provide positive feedback to others, they fail to consider that they might be the recipient of incomplete feedback. As a result, they are left with overconfident self-impressions. Second, social norms lead people to hide their own negative emotional experiences from others. Again, people are aware that this positivity norm influences their own behavior but do not apply this knowledge to their understanding of others. As a result, people regard their own negative emotions as more socially aberrant than is actually the case.
\end{abstract}

Behavior in everyday interactions is powerfully influenced by social norms in the surrounding culture (e.g., Goffman, 1967). For example, social norms exist that encourage the expression of positive rather than negative information within interactions, including positive emotions, experiences, and reactions to others (Eid \& Diener, 2001; Ekman \& Friesen, 1969). As a result, people commonly reply that they are doing "great" or "fine" when asked, even when they have had a terrible day. Similarly, social norms toward positivity lead people to mask many of their more contentious thoughts with politeness (Brown \& Levinson, 1987). After a friend's imperfect piano recital, we might keep our critiques to ourselves and, instead, imply that we enjoyed the performance immensely. Of course, norms encouraging positivity yield a number of benefits within interactions. That said, a central theme of this review is that these benefits carry with them a cost in terms of predictable bias in self and social judgment.

First, we argue that social norms toward positivity lead people to hold incomplete and biased perceptions of others. To the degree that social norms encourage people to share or even exaggerate their positive emotions, experiences, and reactions to partners in social interactions, others' perception of them will be biased toward positive information. Because social norms also encourage people to minimize or hide negative emotions, experiences, and reactions (e.g., Brown \& Levinson, 1987; Eid \& Diener, 2001), this negative information remains invisible and, we suggest, underrepresented in other people's perceptions. As a result, the perceptions people form of others are likely to be predictably biased and over-represent the frequency of others' positive emotions, experiences, and reactions. We argue, further, that these biased perceptions of others contribute to predictable biases in perceptions of the self. 
Our review builds upon past research suggesting that people's perceptions of the internal states of others are generally guided by those cues that are most visible (e.g., Funder, 2001; Watson, Hubbard, \& Wiese, 2000). We review recent research identifying two broad categories of negative information that remain invisible within social interactions. We first review evidence that people keep negative thoughts and reactions to interaction partners hidden in ways that lead those partners' self-assessments astray. Social norms encourage positivity and politeness and, as such, people refrain from giving negative feedback to others and instead provide positive feedback, even when it is not merited. The evidence we review suggests that this tendency for interaction partners to be polite more often than honest contributes to overconfidence when people evaluate themselves.

In a second section, we review evidence that people keep negative emotions hidden in ways that lead interaction partners' self and social judgments astray. Social norms encourage sharing more positive than negative emotions and experiences. Recent research suggests that these norms lead people to underestimate the frequency with which others experience negative emotions. As a result, people can be left feeling that their own negative emotions are less common and "normal" than is actually the case. Across both sections, we highlight the frequency with which negative information remains invisible within interactions, and the consequences that this invisibility has for self and social judgment.

\section{Social Norms and the Self-Assessment of Abilities}

People routinely hold overconfident perceptions of their own traits and abilities (e.g., Alicke, Klotz, Breitenbecher, Yurak, \& Vredenburg, 1995; Dunning, Griffin, Milojkovic, \& Ross, 1990). For example, on average, high school seniors rate themselves in the top quartile in their ability to get along with others, athletic ability, and leadership relative to their peers (College Board, 1976-1977). Gun owners demonstrate overconfidence in their understanding of guns and gun safety (Ehrlinger, Johnson, Banner, Dunning, \& Kruger, 2008). Indeed, Borkenau and Liebler (1993) demonstrated that college students were less accurate in assessments of their own intelligence than were strangers basing their judgments on just 90 seconds of video.

Past research has focused primarily on identifying cognitive (e.g., Dunning, Johnson, Ehrlinger, \& Kruger, 2003; Ehrlinger \& Dunning, 2003) and motivational (e.g., Guenther \& Alicke, 2010; Kunda, 1990; Sedikides, Berbst, Hardin, \& Dardis, 2002) intrapersonal factors that contribute to overconfidence (see Dunning, 2005, for a review). More recent research offers insight into how the informal feedback that we receive in day-to-day interactions might also introduce bias into self-assessments.

\section{The Impact of Social Norms on the Quality of Social Feedback}

People's self-assessments for many abilities and traits are based, at least in part, on social feedback from others. For example, assessments of whether you are funny, motivational, or a good leader require information about the impact that you have on others. Unfortunately, social norms toward positivity encourage polite behavior in social interactions, including offering positive feedback and keeping any points of contention or negative feedback hidden (e.g., Brown \& Levinson, 1987). Emerging research suggests that this absence of negative information in the feedback received within everyday interactions contributes to overconfident self-views.

Past research indicates that people only rarely offer the type of negative feedback that might be necessary for conversation partners to form accurate views of their abilities. For 
example, Camden, Motley, and Wilson (1984) found that people self-report frequent use of "white lies" to protect conversation partners' views of their own competence and personal taste. Participants reported telling white lies in order to satisfy social pressures for politeness or as standard displays of social tact. Ignoring faux pas or feigning unawareness of another's mistakes allows conversations to progress more comfortably for all participants. These lies are commonplace; when participants were asked to review transcripts of their own meaningful conversations (excluding, for example, casual greetings), they judged less than half of their statements to accurately reflect their feelings (Turner, Edgley, \& Olmstead, 1975).

It is noteworthy that a salient reason that people choose politeness over honesty is to avoid the discomfort of revealing negative evaluations and, consequently, leading interaction partners to hold less-positive self-views. DePaulo and Bell (1996) demonstrated that people offered more positive evaluations of paintings when they expected that their evaluations would be seen by the original artist, compared with a condition in which they expected their evaluation to be seen by only uninvested art students. Even when participants were explicitly asked to give feedback that was honest about disliking elements of the paintings, ostensibly to enhance the learning experience for the artists, those who believed their evaluations would be seen by the artist were more positive than controls. It seems, then, that people are reticent to give negative feedback to others even when negative feedback is desired, systematically ensuring that people receive primarily positive feedback about their work and abilities.

\section{Consequences for Self-Assessment}

The research summarized above suggests that negative information about others' abilities is intentionally hidden during many interactions. At the same time, people are aware of normative pressures to limit the expression of negative feedback. On this basis, people might be expected to recognize that the informal feedback they receive about their own abilities and traits is biased, and, as a result, discount it when making self-judgments. However, to the extent that people do not correct for the degree to which feedback they receive might be biased, self-judgments will be overconfident.

Recent research suggests that the latter case is more common. That is, despite knowledge of positivity norms, the tendency for interaction partners to offer overly positive feedback leads people to hold overconfident views about their success in interactions and overconfident perceptions of their abilities. In one recent study, Fay, Ehrlinger, and Goplen (forthcoming) demonstrated that people hold overconfident perceptions of their persuasive abilities, in part, because interaction partners mask the degree to which a persuasive attempt was unsuccessful. In this study, participants were paired together and asked to discuss one of several controversial issues (e.g., legalization of drugs, capital punishment). One participant was randomly assigned to the role of persuader and asked to try to persuade the other (the persuasion target) of his or her view on the issue. After the interaction, participants were separated and asked to answer a series of questions about the interaction. As expected, participants assigned to the role of persuader were overconfident; they offered more positive ratings of their persuasive ability than did persuasion targets. Persuaders were also overconfident when estimating their persuasion target's view on the controversial issue after the interaction. On average, persuaders estimated their targets' views as more in agreement with their own than was actually the case.

If persuaders' overconfidence stemmed directly from a tendency for persuasion targets to mask disagreement, one would expect that neutral people observing the interaction 
would also overestimate the degree to which the persuasion target agreed with the persuader. To examine this prediction, the researchers asked a third set of participants to watch the interactions videotaped from the persuader perspective. As predicted, observers overestimated the degree to which targets agreed with the persuader just as much as did participants in the persuader role (see Figure 1). One would also expect overconfidence to be greatest among those persuaders and observers who were matched with targets who most cared about remaining polite and avoiding conflict. Indeed, this was exactly the case. The more that persuasion targets suggested that it would be impolite to express disagreement, the more their agreement with the persuader was overestimated. These results suggest that targets of a persuasive appeal mask disagreement in an effort to be polite. To the degree that people take persuader- target agreement as genuine evidence of success in being persuasive, they are likely to walk away from the interaction overconfident.

In a follow-up study, Fay et al. (forthcoming) more directly examined whether people recognize that their own behavior within interactions is constrained by a need to be polite but fail to recognize that the same pressures affect their interaction partners and, consequently, interpret interaction partners' behavior as genuine. When someone tells a joke, it is polite to smile and laugh even if the joke is not funny. Fay et al. (forthcoming) examined whether this polite behavior contributes to overconfident perceptions of one's ability to be funny by asking pairs of participants to tell each other humorous jokes or stories. As predicted, participants gave overly positive evaluations of their selected jokes and of their delivery of those jokes, relative to their partner's assessment. People also reported that they laughed at their partner's jokes in order to be polite but that their partner laughed in ways that were genuine. Additional analyses revealed that the degree to which people were overconfident in their ability to be funny was tied directly to their partners' reports of feigning amusement in order to be polite, suggesting that interaction partners reacted in ways that were misleading and that this contributed to overconfident self-assessments.

Together, these studies suggest that people recognize that their own behavior is influenced by positivity norms in that they choose politeness over honesty and offer incomplete or misleading feedback in social interactions. People understand that this norm helps

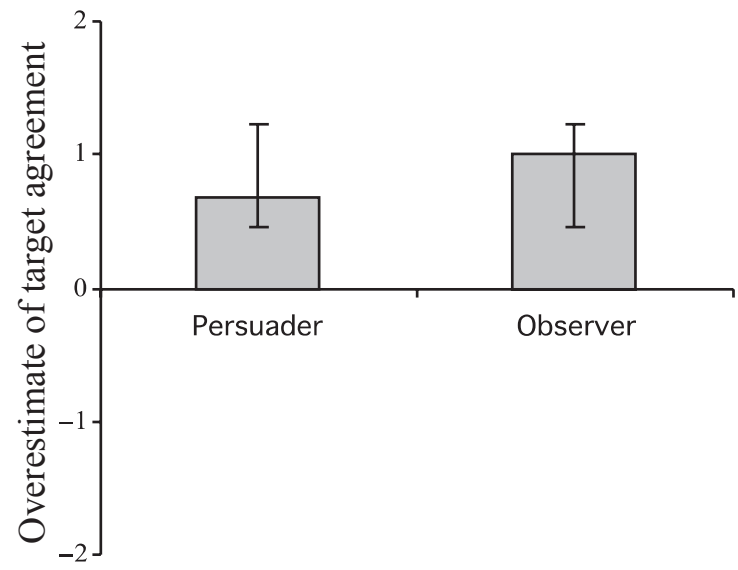

Figure 1 Persuader and observer overestimation of target agreement (Values represent difference scores between estimates of the target view on a 7-point scale and the target's actual view. Graph reproduced from Fay et al., forthcoming.). 
to maintain positive, successful interactions and avoid the confrontation and conflict that might result from offering negative feedback, even when it is honest (Camden et al., 1984). However, when interpreting others' behavior, people fail to apply that understanding of social norms, assuming, instead, that the feedback offered is unbiased. Because positive information is more visible, it outweighs negative information when people make self-assessments. In addition, when receiving feedback about themselves, people may be motivated to accept positive feedback uncritically (e.g., Ditto, Scepansky, Munro, Apanovitch, \& Lockhart, 1998). Consequently, this overly positive feedback, absent important negative information, contributes to overconfident self-views.

\section{Social Norms and the Self-Assessment of Emotional Experience}

Above, we review evidence that negative information about one's traits and abilities is rarely received within social interactions. As a result, people do not have the information necessary to achieve accurate perceptions of the self. We now review evidence suggesting that the invisibility of another type of negative information - information about negative emotions - can leave people with inaccurate perceptions of others and, therefore, erroneous perceptions of their own emotions as abnormal.

Accurate assessment of whether one's emotions are "normal," relative to what others feel, requires an accurate picture of others' emotional lives. However, social norms favor hiding negative emotions and expressing positive emotions. In this section, we review recent research suggesting that people fail to recognize the degree to which these social norms impact the behavior of others and, as a result, perceive negative emotional experiences to be less common than they actually are. The misperception of others' negative emotions may lead people to view their own negative emotions as abnormal, which could in turn exacerbate their negative emotions and, in extreme cases, may even play a role in precipitating psychopathology.

\section{Display Rules and Emotional Pluralistic Ignorance}

All cultures possess social norms about the emotions that are appropriate to display to other people, both in specific situations and in general (Ekman \& Friesen, 1969). Although cultures vary in some aspects of display rules and emotion valuation (Eid \& Diener, 2001), all cultures appear to value happiness and joy positively (Sommers, 1984). In one large study, individuals in American culture were found to place an especially large emphasis on the importance of feeling happiness, joy, love, and pride, to the degree that "being unhappy might be regarded as failing" (Eid \& Diener, 2001, p. 880) within this culture.

Consistent with the high value placed on feeling good (and not feeling bad) in American culture, a study investigating emotion regulation in everyday life among Americans found that the vast majority of emotion regulation attempts were aimed at suppressing or hiding one's negative emotions (Gross, Richards, \& John, 2006). Such emotion regulation occurred almost exclusively in social settings, suggesting that it was aimed at least in part at managing impressions by complying with social norms. Moreover, other investigators have found that college students who recalled recent positive and negative emotional experiences reported that their negative emotional episodes involved efforts to hide their emotions from others at a rate three times higher than their positive emotional episodes did (Jordan, Monin, Dweck, Lovett, John, \& Gross, 2011, Study 1a). Similarly, in the same study, people reported talking less often with peers, after the event, about their negative emotional experiences compared to their positive emotional experiences. 
If negative emotions are preferentially suppressed in public settings, then people may underestimate their actual prevalence, consistent with people's general inaccuracy in judging others' internal experiences in the absence of visible cues (e.g., Funder, 2001; Watson et al., 2000). Recent research suggests that people do in fact underestimate the peer prevalence of negative, but not positive, emotional experiences. In a survey of first-year college students, participants consistently underestimated the commonness of negative emotional experiences among their peers (e.g., having a distressing fight or argument with a friend or partner, or feeling sad because they missed people such as parents or friends), whereas they perceived the commonness of positive emotional experiences accurately, on average, and even overestimated the proportion of their peers who were going out to parties and having fun with friends (Jordan, Monin, et al. 2011, Study 1b). In a second study, students underestimated the extent of negative emotions (e.g., anxiety, loneliness, sadness, anger) in their close friends' lives, whereas they overestimated their close friends' positive emotions (e.g., happiness, pride, connectedness, hope) (Jordan, Monin, et al. 2011, Study 2). Furthermore, students' underestimates of their friends' negative emotions (relative to their positive emotions) were predicted by the degree to which their friends reported preferentially suppressing their negative emotions (relative to their positive emotions). This suggests that the social norms governing emotional displays are so powerful that they even block people from accurately perceiving the emotional lives of well-known others. Although participants in these studies were not asked to directly compare their own emotions to those of their peers, their underestimation of others' negative emotions suggests that they may think they experience more negative emotions themselves than do most other people.

Presumably people are aware that they try to comply with social norms when broadcasting their own emotions to others - playing up the positive, and minimizing the negative - so why do they take other people's happy faces at face value? Indeed, recent research suggests that people who are brokers in social networks - that is, individuals who play a central role in connecting otherwise unconnected people, and who thus normally have greater access to information about social norms within the network - actually make even greater errors in the perception of emotional norms than other individuals (Jordan, Kleinbaum, \& Audia, 2011), suggesting that the more access that people have to others' surface displays of emotion, the more confident they are (incorrectly) that these displays reflect the true reality. This puzzle about people's social inferences applies to all cases of pluralistic ignorance, in which people are blind to the fact that other people's public behaviors are guided by social norms just as much as their own behaviors are, and that their own private experiences are thus actually shared secretly by other individuals more than is apparent from observing social life (Miller \& McFarland, 1987). Drawing on the theorizing of researchers in the pluralistic ignorance tradition, we believe that the misperception of emotional norms likely stems from people's general tendency to underweight situational influences, such as positivity norms, when assessing the causes of others' behaviors (e.g., Ross, 1977).

\section{Consequences of Social Misperceptions and Inaccurate Self-Assessments of Emotions}

The social norm to put positive emotions on display undoubtedly brings some social benefits. For example, emotional well-being has been found to spread within social networks (Hill, Rand, Nowak, \& Christakis, 2010), and this may be due in part to the infectious nature of emotional displays and the influence of such displays on felt emotions (Zajonc, 
Murphy, \& Inglehart, 1989). In addition, given people's tendency to feel emotional empathy more acutely for the negative than for the positive emotions of other people (Royzman \& Kumar, 2001), full social disclosure of negative emotions could lead to an overwhelming sense of empathic sorrow. Furthermore, talking obsessively about one's own negative emotions with other people may be no more healthy than obsessing over the emotions oneself; studies suggest that such co-rumination may be linked to the development of depression (Stone, Uhrlass, \& Gibb, 2010).

At the same time, the norm to express positive emotions and hide negative emotions and the concomitant misperception of actual emotional norms - might lead people to think that their own negative emotions are more socially uncommon and pathological than they really are. Social comparisons in all domains can affect people's well-being (Wheeler \& Miyake, 1992), and upward social comparisons are especially disheartening for people who are already feeling bad (Lyubomirsky \& Ross, 1997; Study 1). Consistent with the notion that (partly illusory) upward emotional comparisons may make people feel worse about themselves, Jordan, Monin, et al. (2011) found that the rarer participants thought that negative emotional experiences were among their peers, the more loneliness and rumination over their own personal problems they reported. Moreover, participants who underestimated peers' negative emotions and overestimated their positive emotions reported feeling less satisfied with their own lives. Of course, these correlations may be due partly to the generation of mood-congruent cognitions (e.g., upward social comparisons) by dysphoric individuals (e.g., Bower, 1991), so interventional studies will be needed to establish causation definitively. Still, recent correlational evidence suggests strongly that the social comparison of emotions may have a serious impact on well-being, with suicide risk greater for individuals who live among exceptionally happy other people, both at the state and the national level (Daly, Oswald, Wilson, \& Wu, 2011).

In addition to feeling worse about oneself due to upward social comparisons - feeling bad about feeling bad, that is - inaccurate assessments of one's own negative emotions as unusual could also lead to attempts to combat those negative emotions. Rather than simply recognizing and accepting that sadness and other emotional challenges are a universal part of life, people who believe these emotions are pathological may be more likely to try to suppress negative thoughts, quash negative emotional responses, and avoid situations that elicit negative emotions. In turn, these individuals might suffer the deleterious psychological and physical health consequences that have been associated with thought suppression (Wegner, 1994; Wenzlaff \& Wegner, 2000), emotional suppression (Gross \& John, 2003; Gross \& Levenson, 1993; Srivastava, Tamir, McGonigal, John, \& Gross, 2009), and avoidance coping strategies (Penley, Tomaka, \& Wiebe, 2002). Indeed, taken to an extreme, attempts to avoid or eliminate negative emotional experience have been theorized to play a role in producing a variety of mental disorders (Hayes, Strosahl, \& Wilson, 1999; Hayes, Wilson, Gifford, Follette, \& Strosahl, 1996; Orsillo \& Roemer, 2005). Innocent attempts to comply with the social norm to express positive emotions, then, may lead to serious and unintended downstream consequences if they cause people to mistakenly perceive their own emotional distress to be abnormal.

\section{Summary and Future Directions}

Because many self-assessments are informed by interactions with others, accuracy in selfassessments often requires accurate perception of others' internal states. However, this review suggests that people's perceptions of others' internal states are often biased because positive information is shared much more freely than negative information within 
interactions. Social norms encourage the expression of information about one's positive emotions and reactions to others, while discouraging the sharing of comparable negative information. Although people seem to understand how positivity norms affect their own behavior within interactions, recent research suggests that they often fail to apply this understanding to the behavior of others. As a result, these normative behaviors introduce error into perceptions of others within interactions and, by extension, perceptions of the self.

This review has focused on two categories of negative information that tend to remain invisible within social interactions - information about negative emotions and about negative reactions to interaction partners. However, we suspect that social norms toward positivity apply to more than just these two categories of information. To the degree that other categories of negative information remain hidden within social interactions, social judgments are likely to be in error, giving too much weight to the positive information that remains visible and too little weight to negative, hidden information. For example, future research might examine whether positivity norms introduce error into perceptions of risk. Just as social norms discourage sharing other types of negative information, we suspect that norms discourage pointing out the risks that people take on a daily basis. It is socially inappropriate, for example, to point out to someone enjoying a cheeseburger or a cigarette that their actions increase their risk of future health problems. Similarly, we tend not to talk about the risks that the years we dedicate working toward academic and career goals might not pay off, that our new relationships might not last forever, or the risks that an upcoming flight or walk across the street might result in our untimely demise. Instead, social norms encourage a focus on positive, optimistic futures within social interactions.

As with other categories of social information, we argue that this focus on the positive can leave people with biased impressions of the level of optimism that others feel and, as a result, mistaken impressions that their own fears about the future are rare. Further, these impressions might leave people feeling that their fears are unfounded and encourage an unrealistic level of optimism. Indeed, people do tend to hold overly optimistic expectations for their future (e.g., Weinstein, 1980). For example, people estimate that they are less at risk of developing a drinking problem, being fired from a job, and attempting suicide than their peers (Weinstein, 1980). To the degree that risk information is hidden in the same way as negative information about abilities, traits, and emotions, this reduced availability of risk information can help to explain why people judge themselves to be less at risk for negative consequences than others.

Future research should explore moderators of the phenomena we have described here. One class of moderators might relate to the type and the strength of social norms present. To the degree that strong social norms exist that result in negative information being invisible and positive information being visible, we would expect the self-assessment biases we discuss in this paper to be exacerbated. Accordingly, we would expect people living in more interdependent cultures to show less biased self-assessments of the commonness of their emotional experiences, compared to people living in more individualistic cultures, because in more interdependent cultural contexts, there is often a social norm to suppress the expression of certain positive emotions (e.g., high-arousal positive emotions) in addition to negative (Gross et al., 2006; Gudyknust, Ting-Toomey, \& Nishida, 1996). This may lead people to form a more balanced view of the valence of others' internal states and more accurately assess the overall quality of others' emotional lives, which in turn would lead to more accurate assessment of one's own emotional wellbeing relative to others'. 
A second class of moderators might relate to differences in the degree to which perceivers accept social normative behavior as unbiased reflections of people's internal states. Here, too, we would expect people in more interdependent cultures to show less of the self-assessment biases we have discussed, since people in more interdependent cultures tend to be less prone to the fundamental attribution error, which may make them more likely to adjust their assessments of others' positive outward expressions according to social normative influences on others' overt behaviors (Miller, 1984; Morris \& Peng, 1994). At the level of individual differences, chronically insecure people may be less likely to accept positive social feedback about themselves as genuine, particularly from partners they know well (Lemay \& Clark, 2008), possibly because they recognize that positive feedback is exaggerated (e.g., Lemay \& Dudley, 2011) or that negative feedback is being concealed. As a result, they may arrive at more accurate self-assessments. Conversely, we would expect highly narcissistic people to show a greater tendency to accept positive social feedback uncritically and even to interpret ambiguous social feedback in a positive light, leading them to more distorted self-assessments (Jordan \& Audia, forthcoming).

Accuracy in self-assessments of abilities, traits, and emotions critically depends on the feedback we receive from others to inform those judgments. Because people have limited access to others' internal states, accurate impressions of others' opinions or experiences require (a) that those opinions are expressed in an unbiased manner, or (b) that people make corrections for biases in the expression of that feedback. Behavior in all social interactions is guided, to some extent, by social norms (Goffman, 1967), so it is unlikely that people regularly receive feedback that is completely unbiased. As such, there is an essential opposition between positivity norms and the pursuit of accurate self-assessments. Accuracy in these judgments, consequently, will be limited by the extent to which diagnostic information is hidden in accordance with positivity norms and people remain un aware of its invisibility. Future research might explore how accuracy in self-assessment could be promoted by a correction of one or both of these issues.

\section{Short Biographies}

Adam Fay's research examines biases in social judgment, the impact of different metaphors for time on attributions of responsibility, and embodied cognition. He earned his BS from Indiana University. He is currently a doctoral student in Psychology at Florida State University, where he recently earned his masters.

Alex Jordan's research centers on people's perceptions of others' emotional lives, social and emotional influences on moral judgment and moral behavior, and the effects of selfenhancement on learning from performance feedback. His work has been published in outlets such as Journal of Personality and Social Psychology, Personality and Social Psychology Bulletin, and Psychological Science. Before beginning his current appointment as a visiting assistant professor at the Tuck School of Business at Dartmouth College, he earned a BA from Harvard University and a PhD in Psychology from Stanford University.

Joyce Ehrlinger's research focuses on understanding the sources of error and accuracy in self and social judgment. For example, Dr. Ehrlinger has examined how self-beliefs regarding ability and regarding the malleability of attributes impact behavior in achievement situations and perceptions of performance. She has also examined how naïve realism leads people to perceive their own views as better informed and less biased than those of others and, in turn, leads to overconfident perceptions of the ease of persuading others. Her work has been feature in a broad range of journals including the Journal of Personality and Social Psychology, the Journal of Experimental Social Psychology, and Personality and Social 
Psychology Bulletin and has been covered in the popular media. She earned her BA at Bowling Green State University and $\mathrm{PhD}$ at Cornell University. After completing a postdoctoral position at Stanford University, Dr. Ehrlinger moved to her current position as assistant professor of psychology at Florida State University. For more information, go to http://www.psy.fsu.edu/ ehrlinger/.

\section{Endnote}

* Correspondence address: Florida State University, 1107 West Call Street, Tallahassee, FL 32306-4301, USA. Email: ehrlinger@psy.fsu.edu

\section{References}

Alicke, M. D., Klotz, M. L., Breitenbecher, D. L., Yurak, T. J., \& Vredenburg, D. S. (1995). Personal contact, individuation, and the better-than-average effect. Journal of Personality and Social Psychology, 68, 804-825.

Borkenau, P., \& Liebler, A. (1993). Convergence of stranger ratings of personality and intelligence with self-ratings, partner ratings, and measured intelligence. Journal of Personality and Social Psychology, 65, 546-553.

Bower, G. H. (1991). Mood congruity of social judgments. In J. P. Forgas (Ed.), Emotion and Social Judgments (pp. 31-53). Elmsford, NY: Pergamon Press.

Brown, P., \& Levinson, S. C. (1987). Politeness: Some Universals in Language Usage. Cambridge, England: Cambridge University Press.

Camden, C., Motley, M. T., \& Wilson, A. (1984). White lies in interpersonal communication: A taxonomy and preliminary investigation of social motivations. Western Journal of Speech Communication, 48, 309-325.

College Board. (1976-1977). Student Descriptive Questionnaire. Princeton, NJ: Educational Testing Service.

Daly, M. C., Oswald, A. J., Wilson, D., \& Wu, S. (2011). Dark contrasts: The paradox of high rates of suicide in happy places. Journal of Economic Behavior and Organization, 80, 435-442.

DePaulo, B. M., \& Bell, K. L. (1996). Truth and investment: Lies are told to those who care. Journal of Personality and Social Psychology, 71, 703-716.

Ditto, P. H., Scepansky, J. A., Munro, G. D., Apanovitch, A. M., \& Lockhart, L. K. (1998). Motivated sensitivity to preference-inconsistent information. Journal of Personality and Social Psychology, 75, 53-69.

Dunning, D. (2005). Self-Insight: Roadblocks and Detours on the Path to Knowing Thyself. New York: Psychology Press.

Dunning, D., Griffin, D. W., Milojkovic, J. D., \& Ross, L. (1990). The overconfidence effect in social prediction. Journal of Personality and Social Psychology, 58, 568-581.

Dunning, D., Johnson, K., Ehrlinger, J., \& Kruger, J. (2003). Why people fail to recognize their own competence. Current Directions in Psychological Science, 12, 83-87.

Ehrlinger, J., \& Dunning, D. (2003). How chronic self-views influence (and potentially mislead) estimates of performance. Journal of Personality and Social Psychology, 84, 5-17.

Ehrlinger, J., Johnson, K., Banner, M., Dunning, D., \& Kruger, J. (2008). Why the unskilled are unaware: Further explorations of (absent) self-insight among the incompetent. Organizational Behavior and Human Decision Processes, 105, 98-121.

Eid, M., \& Diener, E. (2001). Norms for experiencing emotions in different cultures: Inter- and intranational differences. Journal of Personality and Social Psychology, 81, 869-885.

Ekman, P., \& Friesen, W. V. (1969). The repertoire of nonverbal behavior: Categories, origins, usage, and coding. Semiotica, 1, 49-98.

Fay, A. J., Ehrlinger, J., \& Goplen, J. (forthcoming). How Social Norms Contribute to Inaccurate Self-Assessment. Manuscript in Preparation, Florida State University.

Funder, D. C. (2001). Personality. Annual Review of Psychology, 52, 197-221.

Goffman, E. (1967). Interaction Ritual: Essays on Face-to-Face Interaction. New York: Anchor Books.

Gross, J. J., \& John, O. P. (2003). Individual differences in two emotion regulation processes: Implications for affect, relationships, and well-being. Journal of Personality and Social Psychology, 85, 348.

Gross, J. J., \& Levenson, R. W. (1993). Emotional suppression: Physiology, self-report, and expressive behavior. Journal of Personality and Social Psychology, 64, 970-986.

Gross, J. J., Richards, J. M., \& John, O. P. (2006). Emotion regulation in everyday life. In D. K. Snyder, J. Simpson \& J. N. Hughes (Eds.), Emotion Regulation in Couples and Families: Pathways to Dysfunction and Health (pp. 13-35). Washington, D.C.: American Psychological Association.

Gudyknust, W. B., Ting-Toomey, S., \& Nishida, T. (1996). Communication in Personal Relationships across Cultures. Thousand Oaks, CA: Sage Publications. 
Guenther, C. L., \& Alicke, M. D. (2010). Deconstructing the better-than-average effect. Journal of Personality and Social Psychology, 99, 755-770.

Hayes, S. C., Strosahl, K. D., \& Wilson, K. G. (1999). Acceptance and Commitment Therapy: An Experiential Approach to Behavior Change. New York: Guilford.

Hayes, S. C., Wilson, K. G., Gifford, E. V., Follette, V. M., \& Strosahl, K. (1996). Experiential avoidance and behavioral disorders: A functional dimensional approach to diagnosis and treatment. Journal of Consulting and Clinical Psychology, 64, 1152-1168.

Hill, A. L., Rand, D. G., Nowak, M. A., \& Christakis, N. A. (2010). Emotions as infectious diseases in a large social network: The SISa model. Proceedings of the Royal Society B, 277, 3827-3835.

Jordan, A. H., \& Audia, P. G. (forthcoming). Self-enhancement and learning from performance feedback. Academy of Management Review, 37.

Jordan, A. H., Kleinbaum, A. M., \& Audia, P. G. (2011). Social Networks and the Perception of Emotional Norms. Unpublished manuscript. Hanover, NH: Dartmouth College.

Jordan, A. H., Monin, B., Dweck, C. S., Lovett, B. J., John, O. P, \& Gross, J. J. (2011). Misery has more company than people think: Underestimating the prevalence of others' negative emotions. Personality and Social Psychology Bulletin, 37, 120-135.

Kunda, Z. (1990). The case for motivated reasoning. Psychological Bulletin, 108, 480-498.

Lemay, E. P., Jr., \& Clark, M. S. (2008). "Walking on eggshells": How expressing relationship insecurities perpetuates them. Journal of Personality and Social Psychology, 95, 420-441.

Lemay, E. P., Jr., \& Dudley, K. L. (2011). Caution: fragile! Regulating the interpersonal security of chronicallly insecure partners. Journal of Personality and Social Psychology, 100, 681-702.

Lyubomirsky, S., \& Ross, L. (1997). Hedonic consequences of social comparison: A contrast of happy and unhappy people. Journal of Personality and Social Psychology, 73, 1141-1157.

Miller, D. T., \& McFarland, C. (1987). Pluralistic ignorance: When similarity is interpreted as dissimilarity. Journal of Personality and Social Psychology, 53, 298-305.

Miller, J. G. (1984). Culture and the development of everyday social explanation. Journal of Personality and Social Psychology, 46, 961-978.

Morris, M. W., \& Peng, K. (1994). Culture and cause: American and Chinese attributions for social and physical events. Journal of Personality and Social Psychology, 67, 949-971.

Orsillo, S. M., \& Roemer, L. (Eds.) (2005). Acceptance and Mindfulness-Based Approaches to Anxiety: Conceptualization and Treatment. New York: Springer.

Penley, J. A., Tomaka, J., \& Wiebe, J. S. (2002). The association of coping to physical and psychological health outcomes: A meta-analytic review. Journal of Behavioral Medicine, 25, 551-603.

Ross, L. (1977). The intuitive psychologist and his shortcomings: Distortions in the attribution process. In L. Berkowitz (Ed.), Advances in Experimental Social Psychology (vol. 10, pp. 173-220). New York, NY: Academic Press.

Royzman, E. B., \& Kumar, R. (2001). On the relative preponderance of empathic sorrow and its relation to commonsense morality. New Ideas in Psychology, 2, 131-144.

Sedikides, C., Berbst, K. C., Hardin, D. P., \& Dardis, G. J. (2002). Accountability as a deterrent to self-enhancement: The search for mechanisms. Journal of Personality and Social Psychology, 83, 592-605.

Sommers, S. (1984). Adults evaluating their emotions: A cross-cultural perspective. In C. Z. Malatesta \& C. Izard (Eds.), Emotions in Adult Development (pp. 319-338). Beverly Hills, CA: Sage.

Srivastava, S., Tamir, M., McGonigal, K. M., John, O. P., \& Gross, J. J. (2009). The social costs of emotional suppression: A prospective study of the transition to college. Journal of Personality and Social Psychology, 96, 883897.

Stone, L. B., Uhrlass, D. J., \& Gibb, B. E. (2010). Co-rumination and lifetime history of depressive disorders in children. Journal of Clinical Child and Adolescent Psychology, 39, 597-602.

Turner, R. E., Edgley, C., \& Olmstead, G. (1975). Information control in conversations: Honesty is not always the best policy. The Kansas Journal of Sociology, 11, 69-89.

Watson, D., Hubbard, B., \& Wiese, D. (2000). Self-other agreement in personality and affectivity: Effects of acquaintanceship, trait visibility, and assumed similarity. Journal of Personality and Social Psychology, 78, 546-558.

Wegner, D. M. (1994). Ironic processes of mental control. Psychological Review, 101, 34-52.

Weinstein, N. D. (1980). Unrealistic optimism about future life events. Journal of Personality and Social Psychology, 39, 806-820.

Wenzlaff, R. M., \& Wegner, D. M. (2000). Thought suppression. Annual Review of Psychology, 51, 59-91.

Wheeler, L., \& Miyake, K. (1992). Social comparison in everyday life. Journal of Personality and Social Psychology, 62, 760-773.

Zajonc, R. B., Murphy, S. T., \& Inglehart, M. (1989). Feeling and facial efference: Implications for the vascular theory of emotion. Psychological Review, 96, 395-416. 